\section{A device for presenting targets and recording positioning responses in one dimension}

\author{
JOHN J. UHLARIK \\ Kansas State University, Manhattan, Kansas 66502
}

Apparatus for recording manual target pointing localizations is described. The device, utilizing a position transducer and digital voltmeter, is capable of determining the position of either manual limb when localizing visual and/or auditory targets. The pointing localizations involve naturalistic limb movements made in a relatively unrestricted manner. The apparatus has the advantages of rapid accurate measurement, reliability, ease of reading, and the potential for automatic recording. When used for research involving adaptation to sensory rearrangement, there is the further advantage that the dependent measures are obtained in the same context as during exposure to the rearrangement.

It has been previously pointed out (Bauer, Woods, \& Held, 1969) that research on human spatial orientation and sensorimotor coordination often involves pointing at a target or some arbitrary referent such as "straight ahead." The responses involved are pointing localizations made with the manual limb. The appropriate measure of this response is the position of the tip of the index finger relative to the position of the target.

The apparatus that is presented in this paper was developed to conduct research in the area of prism adaptation. It has several advantages when compared to techniques currently employed, and the device could find application in a number of different research methodologies involving localizations of visual, auditory, or other types of spatial referents. The targets are easily presented, and the measures are rapid, reliable, and accurate. The localizations entail naturalistic pointing responses that can be made with either limb.

The apparatus is illustrated in Fig. 1. It consists of a horizontal panel mounted on top of a table. The $S$ is seated facing the apparatus and makes localizing responses by reaching under the horizontal panel. This panel has an opening, illustrated in Fig. 2, that permits $S$ to view his limb while making localizations. The opening can be covered when it is necessary to preclude visual feedback from the pointing limb. The advantage of this arrangement when used in the typical prism-adaptation paradigm is that it allows the dependent measures to be obtained in the same place and under conditions that are as similar as possible to those present during exposure to rearrangement. The essential difference between the dependent measures and exposure is that visual feedback from the pointing limb is usually allowed only during exposure.
The target display consists of a vertical panel that is perpendicular to S's line of sight. This panel is approximately $56 \mathrm{~cm}$ from the frontal surface of S's cornea and curved to a radius of $57 \mathrm{~cm}$. Thus, 1 linear $\mathrm{cm}$ on the panel subtends a visual angle of approximately $1 \mathrm{deg}$. The panel is constructed of a metal frame covered with black felt cloth that permits either transillumination with a movable target light or the presentation of an auditory target by means of a movable speaker. Both the rubber foam so that they will not provide extraneous auditory cues during movement. These targets can be transported on a track that is also curved to the arc of the stimulus display. This arc is located $3 \mathrm{~cm}$ behind the vertical panel of the stimulus display so that the targets can target and speaker are mounted on

be presented in a horizontal plane at S's eye level. In addition, there are 61 fixed target lights on the display panel. Each light is $2 \mathrm{~mm}$ in diam, spaced $1 \mathrm{~cm}$ apart on center, and can be individually illuminated by either $\mathbf{S}$ or $E$ with a stepping switch. The $S$ views the target display with his head held in a fixed position by a biteboard containing his dental impression. All surfaces of the apparatus are covered with black felt cloth to eliminate any reflections of incident light.

The position transducer consists of a Plexiglas rod, $1.27 \mathrm{~cm}$ in diam and $1 \mathrm{~m}$ in length. The rod is threaded with a die that cuts approximately 8 threads $/ \mathrm{cm}$. Resistance wire, such as No. 26 B \& S nichrome, is wound in a coil using the threads of the rod. The resistance of this particular gauge wire

is $8: 44 \mathrm{ohms} / \mathrm{m}( \pm 1 \%)$. The entire coil is curved to a radius of $57 \mathrm{~cm}$ and mounted on the side of the vertical display panel facing $S$ (see Fig. 1). Thus, the position transducer will measure over a range in the horizontal plane that would subtend a visual arc equivalent to $100 \mathrm{deg}$. The actual measures are obtained by having $S$ complete an electrical circuit by touching a stylus to the position transducer. This circuit is shown in Fig. 3. By completing the circuit with the stylus attached to S's index finger, the coil provides an electrical signal that is linearly proportional to the position of the stylus on the coil. This signal is measured and registers on a digital display voltmeter in the circuit. The range of this particular meter is $0-1.999 \mathrm{~V}( \pm 0.1 \%)$ and is sufficiently accurate to read reliably differences as

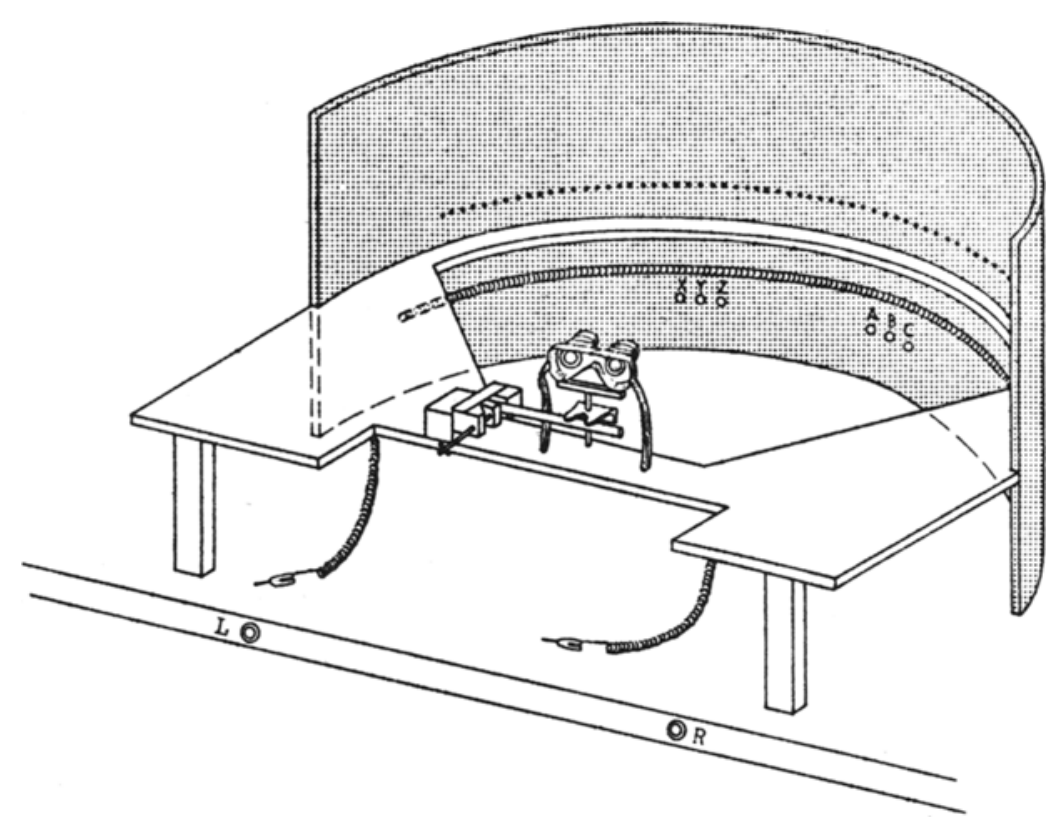

Fig. 1. Perspective view of the apparatus. 


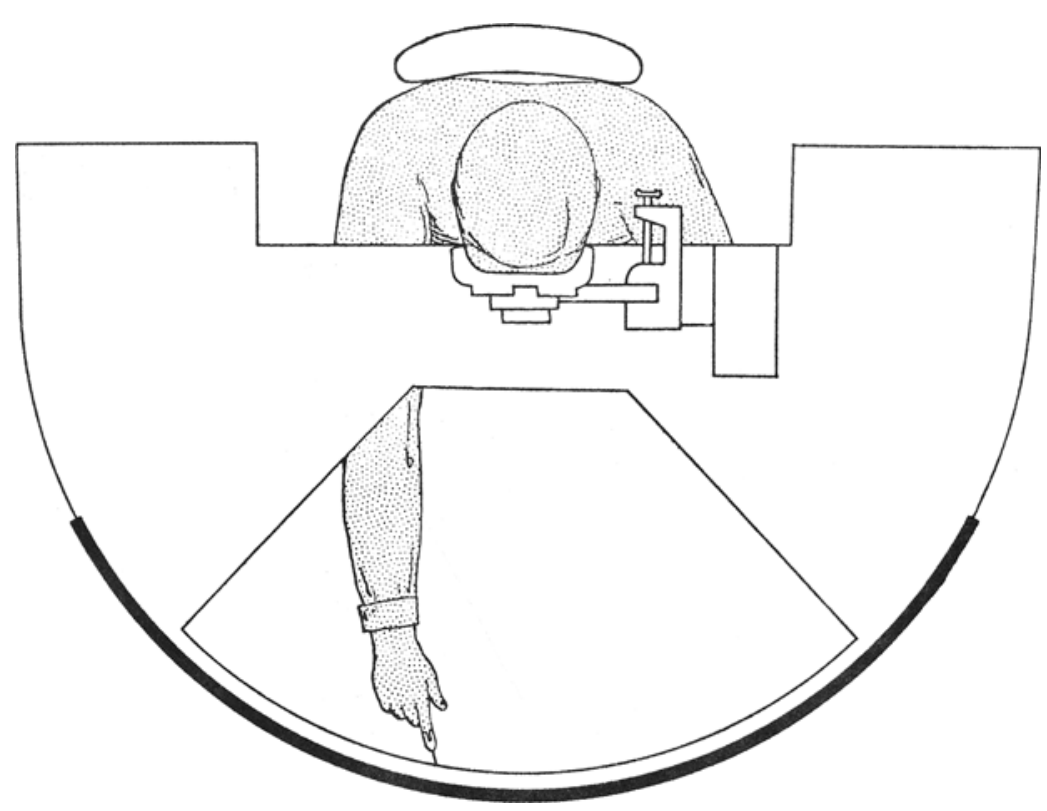

Fig. 2. Top view of apparatus during exposure.

small as those obtained between single windings on the coil. In this case the readings are accurate to the nearest $.13 \mathrm{deg}$. The digital display is easily read and is not prone to errors in reading as are moving coil meters. Furthermore, $\mathrm{E}$ can cause the meter

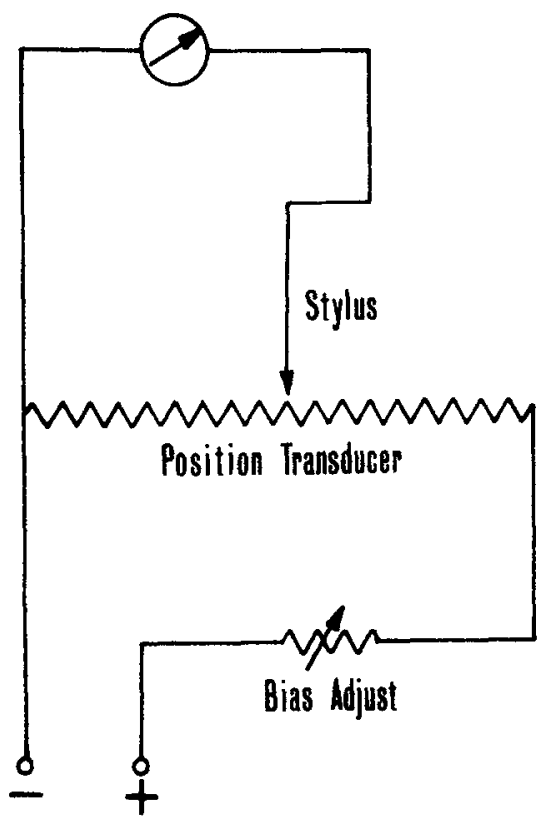

Fig. 3. Electrical diagram for metering circuit and position transducer. his limb to such referents conjunction with a pivoting biteboard, it is possible to measure similar localizations made with the head and neck relative to the rest of the body.

All of the localizations can be made very naturally and comfortably by Ss. A minimum of instructions and pretraining is required to obtain reliable and accurate measures of sensorimotor orientation.

The techniques for measuring positioning responses employed by Held and his coworkers (e.g., Held \& Gottlieb, 1958; Bauer, Woods, \& Held, 1969) allow the recording of these responses in two dimensions. The apparatus just described allows both the presentation of targets and the recording of positioning responses in only one dimension. There are several advantages of this system relative to the ones developed by Held and associates. The position transducer and target array are curved in a horizontal plane relative to $S$. Thus, the targets are presented at eye level so that visual targets of a fixed size subtend the same visual angle at all points on the arc, and both auditory and visual targets are always equidistant from the center of S's "cyclopian eye." If, for example, a visual target of a fixed size was presented and localized in a display located in S's frontoparallel plane, as would undoubtedly be the case in an apparatus recording in two dimensions, the visual angle and the shape of the target would vary as a function of the distance from S's midsagittal plane.

A second advantage of the present apparatus is that the targets to be localized are in the same orientation relative to $S$, as is the position transducer. Thus, there is no need to localize virtual images reflected in a mirror, and it allows the presentation of auditory targets. Thirdly, the position transducer used by Bauer et al (1969) is reported to be accurate to the nearest 10 th of an inch. In the present system, the transducer yields positions that correspond to the nearest 20th of an inch. "straight ahead," and to determine, independently of the felt position of the limbs, which positions of the light and sound appear to be "straight ahead." The E merely has to press switches, move the targets, and record the data. All of these functions can be performed while $E$ is seated out of $S$ 's field of view. Furthermore, by using a position transducer such as a micropotentiometer that rotates in
REFERENCES

BAUER, J. A., WOODS, G. W., \& HELD, R. A device for rapid recording of positioning responses in two dimensions. Behavior Research Methods \& Instrumentation, 1969, 1, 157-159.

HELD, R., \& GOTTLIEB, N. A technique for studying adaptation to disarranged hand-eye coordination, Perceptual \& Motor Skills, 1958, 8, 83-86. 